\title{
Comparison of Software Decision Support Systems for Solving a Multicriteria Optimization Problem
}

\author{
Boris Staykov, Vladimir Monov \\ Institute of Information and Communication Technologies, Bulgarian Academy of Sciences, 1113 Sofia, \\ Bulgaria \\ E-mails: bstaykov@iinf.bas.bgvvmonov@iit.bas.bg
}

\begin{abstract}
This article describes in details how multicriteria optimization can be applied to solve a typical business problem for resources planning and manufacturing process optimization in a battery factory. We solve the problem by using an interactive software decision support system WebOptim developed at the Institute of information and communication technologies. The entire problem solving process is described step by step in order to point out the problem specific features as well as to demonstrate the capabilities of the WebOptim software system. For comparison, we have solved the same problem by means of another popular decision support system WWW NIMBUS and both solutions are analyzed and discussed.
\end{abstract}

Keywords: Multicriteria optimization, decision support systems, resource planning.

\section{Intoduction}

In nowadays business and economy, many problems in complex systems management and control, planning, analysis and monitoring, can be described and solved as multicriteria decision-making problems $[1,16,17,19]$. These problems on the other hand can be divided into two large classes $[2,3]$, depending on their formal statement.

First class contains a finite number of explicitly set constraints as functions. These functions define an infinite number of feasible alternatives [4]. These are so called continuous multicriteria optimization problems.

In the second class of problems, there is finite number of alternatives, described in tabular form [5]. These problems are called discrete multicriteria decision making problems or multicriteria analysis problems.

In this paper the focus is on the first class - multicriteria optimization problems. Their specific characteristics are briefly described as follows.

Multicriteria optimization problems are non-formalized or weakly formalized, the solution of which requires active participation of the so-called Decision Maker (DM) $[6,7]$. Usually, solutions that are obtained depend very much on the DM's 
personal preferences. In multicriteria optimization problems, several criteria (objective functions) are simultaneously optimized into a feasible set of alternatives. Normally a single alternative does not exist that optimizes all the criteria. However, a small subset of alternatives exist, where each improvement in the value of one criterion, leads to deterioration in the value of at least one other criterion. Each solution from this subset is called Pareto-optimal solution [8]. Therefore, when we speak about multicriteria optimization, we speak about finding a good Pareto-optimal solution. What is a "good solution" is a decision, taken by the decision maker.

Two main approaches are used to solve multicriteria optimization problems: scalarizing approach [9] and approximation approach [10]. The scalarizing approach is more popular. In that case, the multicriteria optimization problem is reduced to a single-criteria optimization problem. This approach uses interactive algorithms, where the decision maker has the most important role in the solution finding process. Each interactive algorithm contains two steps - optimization and evaluation, which are repeated in cycle until a final decision is made. In the evaluation step, the decision maker evaluates the current Pareto-optimal solution. If it satisfies his personal preferences the process stops and that current solution becomes a final one. In the other case, the decision maker defines certain preferences concerning changes in criteria values in order to obtain a new Pareto-optimal solution. Based on those preferences in the optimization step a new scalarizing problem is generated and solved, which gives the new consecutive Pareto-optimal solution. As mentioned before - the scalarizing problem is a single-criteria optimization problem, which makes possible using the well-known theory and algorithms for solving singlecriteria optimization problems.

Many interactive algorithms are developed, each one heaving advantages and disadvantages, concerning the type of information that the decision maker provides as a reflection of his personal global and local preferences.

The most important aspect in multicriteria optimization is that the process involves heavy mathematical calculations repeated in multiple iteration cycles. Those calculations are practically impossible to make without the help of so-called decision support systems. They are software systems that provide the decision maker with the tools and ability to solve such problems by implementation of the two steps optimization and evaluation. These systems are classified in two classes - software systems with general purpose and problem-oriented software systems.

The general-purpose software systems aid the process of finding an optimal solution by different decision makers with different level of experience and knowledge. The systems usually implement one method or several methods from the same group.

Problem-oriented software systems are part of other software systems and serve to aid the solution of one or several types of specific multicriteria optimization problems. Specialized methods and algorithms are also developed in the cases of group and fuzzy multicriteria decision making [20, 21].

Well-known general-purpose software systems, which support the solving of multicriteria optimization problems, are VIG, DIDAS, DINAS, MOLP-16, LBS, SOMMIX, MOIP, WWW-NIMBUS [15], MOLIP, NLPJOB and MOMILP [11,12]. 
This article demonstrates how to solve a real problem for production planning by using the software decision support system WebOptim [13]. It is an interactive software system for multicriteria optimization, developed at the Institute of information and communication technologies, Bulgarian Academy of Sciences. It implements some of the most well-known algorithms, and one interactive algorithm developed at the same institute [14]. The first author of this article is one of the leading developers of the WebOptim software system. The system is contemporary, user friendly for many users and provides the decision maker with a large variety of instruments to solve multicriteria optimization problems [18].

\section{Problem description}

The problem under consideration is a benchmark example taken from a book with case studies of typical multicriteria optimization problems [17] and it is adapted for the demonstration purposes of this article. The problem description is as follows.

A Battery fabric is manufacturing three types of batteries for selling on internal and international market:

- Standard capacity battery

- High capacity battery

- Rechargeable battery

The manufacturing process defines certain requirements and dependencies between manpower and machine power for a specific period of time. These dependencies are shown in Table 1.

Table 1. Resources and prices

\begin{tabular}{|l|c|c|c|c|}
\hline Parameter & $\begin{array}{c}\text { Manpower } \\
\text { hours }\end{array}$ & $\begin{array}{c}\text { Machine } \\
\text { power hours }\end{array}$ & $\begin{array}{c}\text { Price on } \\
\text { internal market }\end{array}$ & $\begin{array}{c}\text { Price on international } \\
\text { market }\end{array}$ \\
\hline Standard capacity battery & 32 & 19 & 340 & 350 \\
\hline High capacity battery & 38 & 21 & 362 & 368 \\
\hline Rechargeable battery & 39 & 23 & 374 & 378 \\
\hline Available resources & 16,000 & 9,000 & & \\
\hline Resource price & 4 & 2.5 & & \\
\hline
\end{tabular}

Goal: To optimize the manufacturing process: resources; prices; profit.

Initial Constraints:

Company policy requires selling each battery type on both markets - internal and international. There are also requirements concerning the minimum quantities of each battery type units. They are as follows:

- Minimum 30 units of standard capacity batteries

- Minimum 20 units of high capacity batteries

- Minimum 10 units of rechargeable batteries

Varaibles:

- ib1 - standard capacity batteries quantity for internal market

- eb1 - standard capacity batteries quantity for international market

- ib2 - high capacity batteries quantity for internal market 
- eb2 - high capacity batteries quantity for international market

- ib3 - rechargeable batteries quantity for internal market

- eb3 - rechargeable batteries quantity for international market

- r1 - variable specifying the constraint of manpower resources

- r2 - variable specifying the constraint of machine power resources

Objective functions:

- Maximize the profit F1 (manufacturing price-expenses) -

$\operatorname{MaxF} 1=164.5 \times \mathrm{ib} 1+174.5 \times \mathrm{eb} 1+157.5 \times \mathrm{ib} 2+163.5 \times \mathrm{eb} 2+160.5 \times \mathrm{ib} 3+$ $+164.5 \times \mathrm{eb} 3$,

where each coefficient is the difference between the selling price and the manufacturing expenses defined with the following formulae:

Coefficient of $\mathrm{ib} 1=340-(4 \times 32+2.5 \times 19)=164.5$;

Coefficient of eb1 $=350-(4 \times 32+2.5 \times 19)=174.5$;

Coefficient of $\mathrm{ib} 2=362-(4 \times 38+2.5 \times 21)=157.5$;

Coefficient of eb2 $=368-(4 \times 38+2.5 \times 21)=163.5$;

Coefficient of ib3 $=374-(4 \times 39+2.5 \times 23)=160.5$;

Coefficient of eb3 $=378-(4 \times 39+2.5 \times 23)=164.5$.

Next task is to optimize the using of manpower and machine power resources. This is done through minimizing the difference between the total available resource and the sum of all resource hours necessary for the manufacturing process.

- Minimizing manpower resource F2:

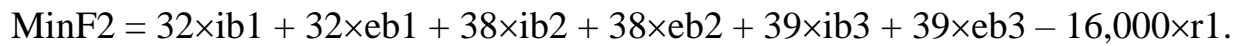

- Minimizing machine power resource F3:

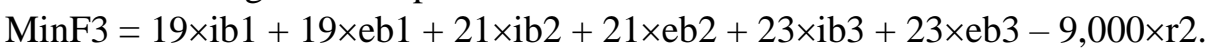

Constraints:

- Manpower resource constraint: $32 \times \mathrm{ib} 1+32 \times \mathrm{eb} 1+38 \times \mathrm{ib} 2+38 \times \mathrm{eb} 2+39 \times \mathrm{ib} 3+39 \times \mathrm{eb} 3<=16,000$.

- Machine power constraint: $19 \times \mathrm{ib} 1+19 \times \mathrm{eb} 1+21 \times \mathrm{ib} 2+21 \times \mathrm{eb} 2+23 \times \mathrm{ib} 3+23 \times \mathrm{eb} 3<=9,000$.

After a solid business analysis, managers have settled minimum battery quantity requirements for internal and external market as follows:

ib $1 \geq 40$

eb1 $\geq 40$

ib $2 \geq 40$

eb2 $\geq 30$

ib3 $\geq 20$

eb3 $\geq 20$

\section{Solving the problem and making a decision with WebOptim}

After entering the problem definition in the WebOptim decision support system (Fig. 1), we obtain auto generated first initial solution (Fig. 2). 


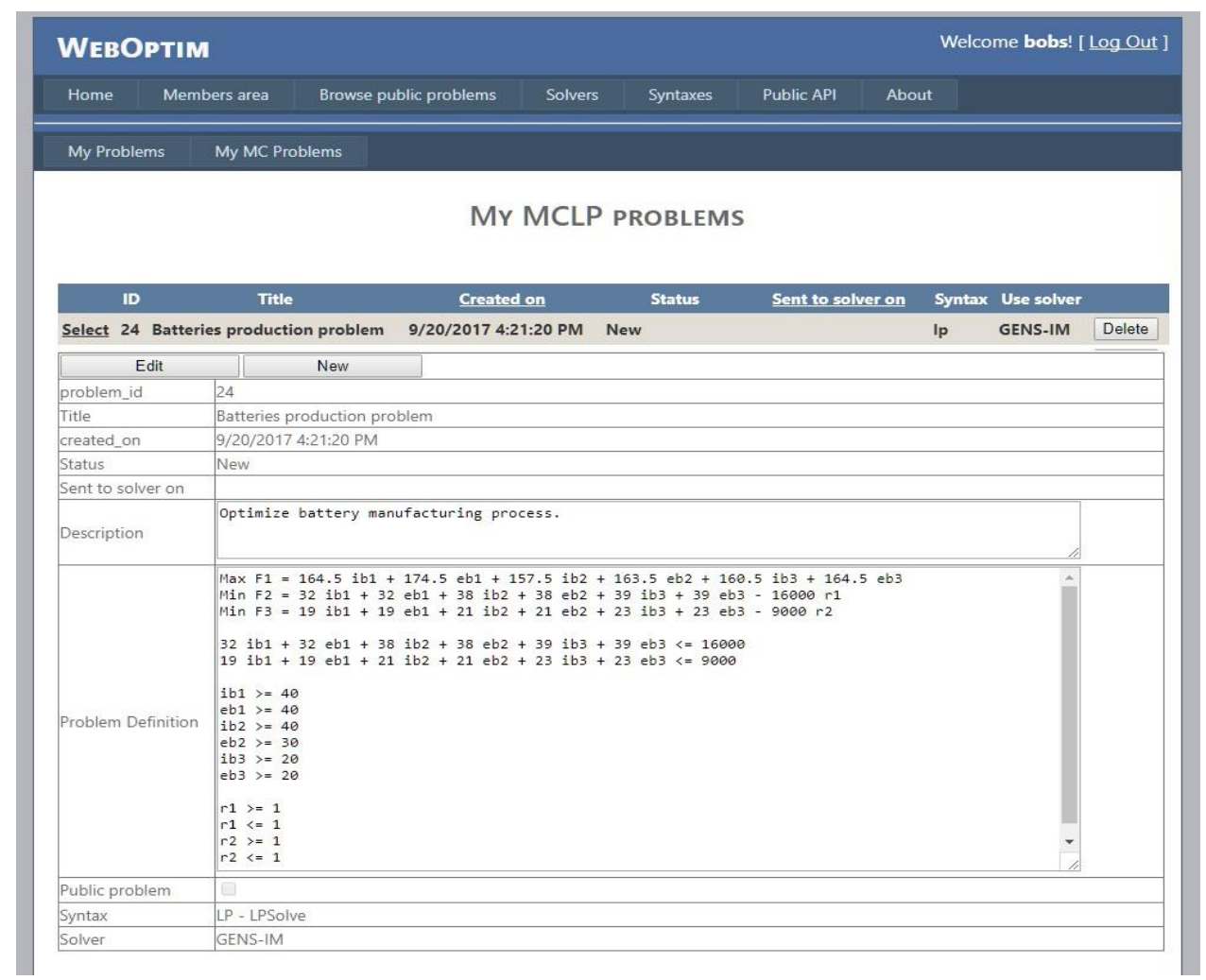

Fig. 1. WebOptim system - problem definition interface

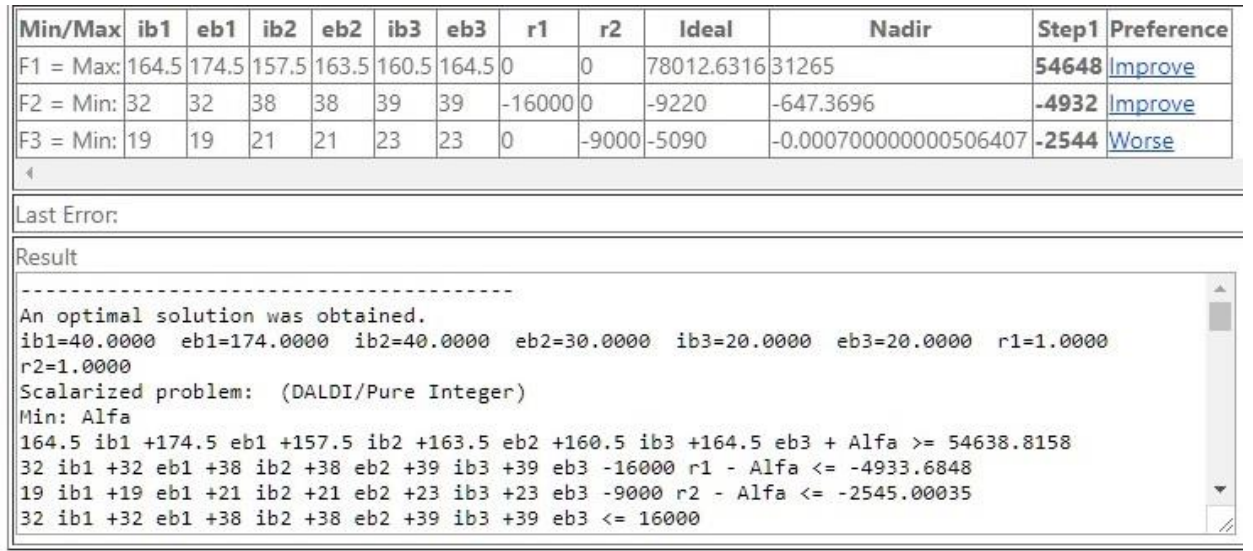

Fig. 2. WebOptim system - initial solution and preferences set for the next step

Result values related to batteries quantity are given in Table 2 and objective function values are in Table 3.

Table 2. Battery quantity - initial values

\begin{tabular}{|l|c|c|c|}
\hline Market & Standard capacity & High capacity & Rechargeable \\
\hline Internal & 40 & 40 & 20 \\
\hline International & 174 & 30 & 20 \\
\hline
\end{tabular}


Table 3. Objective function initial values

\begin{tabular}{|c|c|c|}
\hline F1 & F2 & F3 \\
\hline 54,648 & $-4,932$ & $-2,544$ \\
\hline
\end{tabular}

The first conclusion is that here we have optimized the resources (F2 and F3), but the profit (F1) seems to be unsatisfying. From mathematical viewpoint, this solution is Pareto-optimal, but in business terms, we could ask for more. Here comes the decision maker's active role. It is obvious that the highest profit comes after selling on international market. In our case, the battery quantities for international market are too low. In order to improve the value of objective function F1 (profit), we have to choose which other criterion to worsen and how to worsen. The manager's decision is to worsen the objective function related to machine power resources (F3), because they are cheaper. On the other hand, if we just set preferences to worsen this value, it might go right to its bottom point and we do not want that.

However, as a beginning, we would like to do exactly that - worsen the use of resources, just to see what values will come when maximizing the profit. Later we will do some more changes.

After choosing free improvement of F1 and free worsening of F2 and F3, we obtain the next values shown in Fig. 3, Table 4 and Table 5.

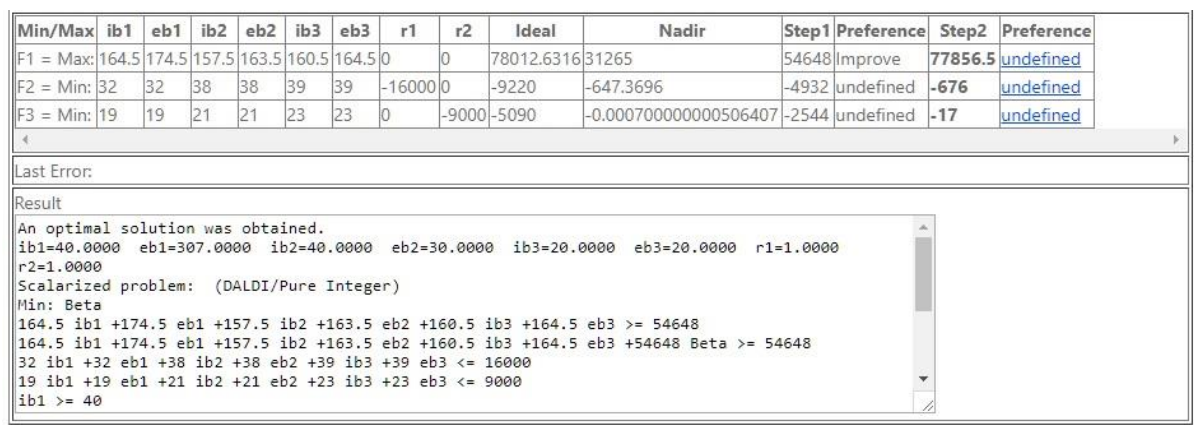

Fig. 3. WebOptim system - second step results

Table 4. Battery quantity - Step 2 values

\begin{tabular}{|l|c|c|c|}
\hline Market & Standard capacity & High capacity & Rechargeable \\
\hline Internal & 40 & 40 & 20 \\
\hline International & 307 & 30 & 20 \\
\hline
\end{tabular}

Table 5. Objective function - Step 2 values

\begin{tabular}{|c|c|c|}
\hline F1 & F2 & F3 \\
\hline $77,856.5$ & -676 & -17 \\
\hline
\end{tabular}

As expected - we have maximized the quantity of standard capacity batteries for export, because they have the highest profit rate. Again as expected - the difference in resources usage functions values is too big compared to the initial solution (Fig. 4). Now we have to optimize that too. Manpower resources (F2) are expensive and we would like to keep them at better value. From the current point, we choose to improve that value with aspiration level of at least $-6,000$. 
Machine power resources (F3) are cheaper and we choose to set their value to be improved to aspiration level of at least $-1,500$. And in order to control the worsening of F1 function, we choose to worsen it to aspiration level of no more than 40,000 (Fig. 4).

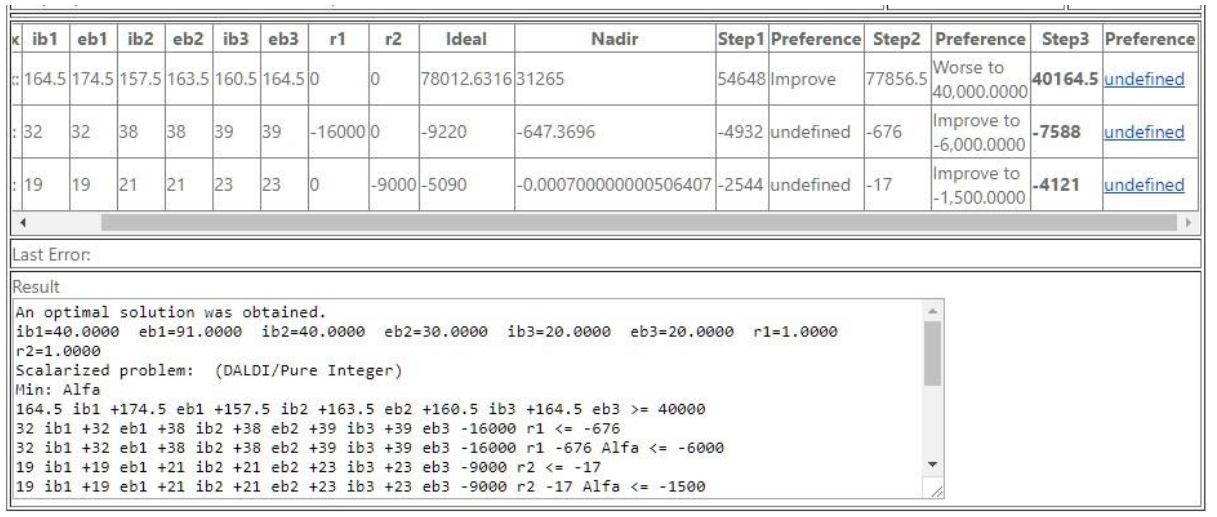

Fig. 4. WebOptim system - third step results

After solving the new problem, we obtain the values given in Tables 6 and 7 .

Table 6 . Battery quantity - Step 3 values

\begin{tabular}{|l|c|c|c|}
\hline Market & Standard capacity & High capacity & Rechargeable \\
\hline Internal & 40 & 40 & 20 \\
\hline International & 91 & 30 & 20 \\
\hline
\end{tabular}

Table 7. Objective function - Step 2 values

\begin{tabular}{|c|c|c|}
\hline F1 & F2 & F3 \\
\hline $40,164.5$ & $-7,588$ & $-4,121$ \\
\hline
\end{tabular}

At that point, we are satisfied with the value of the objective function that is related to manpower resources and we would like to keep it as it is. The profit can be improved more on the account of the cheaper machine resources. In order to improve $\mathrm{F} 1$, we choose to worsen F2 to a level of $-5,000$ and worsen F3 to a level of $-1,500$. After solving, we obtain the values given in Tables 8 and 9 .

Table 8. Battery quantity - final step values
\begin{tabular}{|l|c|c|c|}
\hline Market & Standard capacity & High capacity & Rechargeable \\
\hline Internal & 40 & 40 & 20 \\
\hline International & 171 & 30 & 20 \\
\hline
\end{tabular}

Table 9. Objective function - final step values

\begin{tabular}{|c|c|c|}
\hline F1 & F2 & F3 \\
\hline $54,124.5$ & $-5,028$ & $-2,601$ \\
\hline
\end{tabular}

Our decision is to keep those values as a final solution, where we guarantied the maximum profit and optimal resources usage. 


\section{Solving the problem with Nimbus}

To compare the problem solving process, obtained results and overall user experience, we choose to solve the same task with another popular decision support system that solves similar problems - WWW NIMBUS (wwwnimbus.it.jyu.fi) [15].

The input interface of Nimbus is presented on Fig. 5.

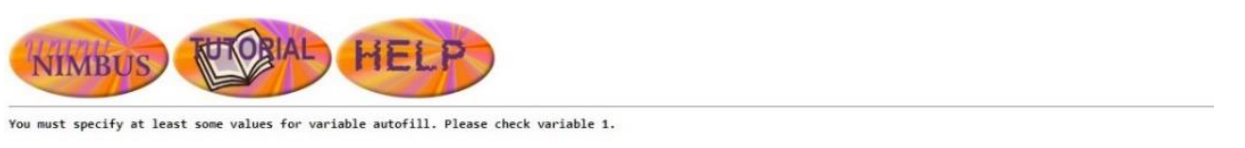

\section{Input Problem}

Fill in all the fields, please. Note that the function and the variable names are used when displaying results, and the variable names $\mathrm{x1}, \mathrm{x} 2, \mathrm{x} 3$, etc. must be used when defining the problem.

Objective functions to be optimized

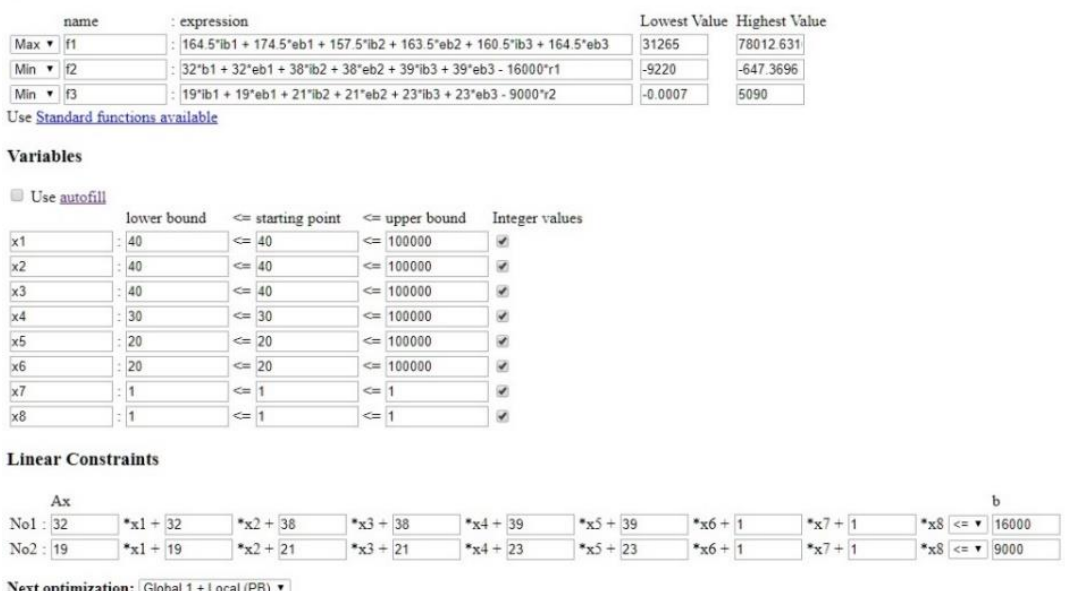

Fig. 5. Nimbus system - problem definition interface

The initial solution that is generated from Nimbus has exactly the same values as this from WebOptim. After two more iterations with similar preferences, the obtained results are given in Table 10.

Table 10. Comparison between obtained solutions from WebOptim and WWWNimbus by steps

\begin{tabular}{|c|l|c|c|c|c|c|c|c|c|c|}
\hline Step & System & ib1 & eb1 & ib2 & eb2 & ib3 & eb3 & F1 & F2 & F3 \\
\hline 1 & WebOptim & $\mathbf{4 0}$ & $\mathbf{1 7 4}$ & $\mathbf{4 0}$ & $\mathbf{3 0}$ & $\mathbf{2 0}$ & $\mathbf{2 0}$ & $\mathbf{5 4 , 6 4 8}$ & $\mathbf{- 4 , 9 3 2}$ & $\mathbf{- 2 , 5 4 4}$ \\
\hline 1 & Nimbus & 40 & 174 & 40 & 30 & 20 & 20 & $54,648.0$ & $-4,932$ & $-2,544$ \\
\hline 2 & WebOptim & $\mathbf{4 0}$ & $\mathbf{9 1}$ & $\mathbf{4 0}$ & $\mathbf{3 0}$ & $\mathbf{2 0}$ & $\mathbf{2 0}$ & $\mathbf{4 0 , 1 6 4 . 5}$ & $\mathbf{- 7 , 5 8 8}$ & $\mathbf{- 4 , 1 2 1}$ \\
\hline 2 & Nimbus & 40 & 40 & 40 & 30 & 20 & 20 & 31,265 & $-9,220$ & $-5,090$ \\
\hline 3 & WebOptim & $\mathbf{4 0}$ & $\mathbf{1 7 1}$ & $\mathbf{4 0}$ & $\mathbf{3 0}$ & $\mathbf{2 0}$ & $\mathbf{2 0}$ & $\mathbf{5 4 , 1 2 4 . 5}$ & $\mathbf{- 5 , 0 2 8}$ & $-\mathbf{2 , 6 0 1}$ \\
\hline 3 & Nimbus & 40 & 174 & 40 & 30 & 20 & 20 & $54,648.0$ & $-4,932$ & $-2,544$ \\
\hline
\end{tabular}

It is seen from the results in Table 10, that at the first step of optimization both software systems WebOptim and Nimbus give exactly the same Pareto-optimal solution. However, applying the specific decision maker's preferences described in the previous section, we obtain different alternatives in the next two steps. 
In particular, at Step 2 the solution obtained by WebOptim is characterized by a substantially better value of the obtained profit (objective function F1) and relatively worse values of the manpower and machine power resources (objective functions F2 and F3) as compared to the respective values in the solution of the Nimbus system. At this step it can be concluded that both solutions are acceptable as they satisfy all constraints of the optimization problem and the decision maker's preferences. Thus, the decision maker can chose one of them depending on some additional economic preferences and/or production process specifics.

At Step 3 it is seen that the solution obtained by Nimbus actually repeats the initial Pareto-optimal solution. At the same time the WebOptim system produces a solution which is characterized by a slightly worse value of the objective function F1 and better values of objective function F1 and F2. Thus at this step the software system WebOptim provides another more alternative which gives the decision maker more possibilities to find the most appropriate final decision.

\section{Conclusion}

In this work we presented and analysed in details the solving process of a multicriteria optimization problem and the corresponding decision making for an efficient resources planning and production process optimization in a battery manufacturing company. The entire solving process involves three main components: mathematical model of the production process and business requirements, specialized software implementing algorithms for multicriteria optimization and the active participation of the decision maker. We have used an interactive decision support system WebOptim in order to find a Pareto optimal solution of the problem together with several alternative solutions corresponding to different preferences of the decision maker. For comparison, the same task is solved with another software system WWW Nimbus supporting the decision making in similar problems. An analysis of both solutions indicates that WebOptim is advantageous in providing more alternatives for making the most appropriate final decision. In a future perspective, it would be useful to develop and implement new exact and heuristic algorithms for solving wider range of complex optimization problems. Finally, as a general conclusion it should be noted that real life decision making problems are difficult to solve and only the combination of exact mathematical model, efficient software tools and active participation of the decision maker is a guarantee for finding a scientifically justified solution.

\section{References}

1. E o m, S. B., S. M. L e e, E. B. K i m, C. S o m a r a j a n. A Survey of Decision Support System Applications (1988-1994). - Journal of Operational Research Society, Vol. 49, 1998, No 2, pp. 109-120.

2. S t e u e r, R. Multiple Criteria Optimization: Theory, Computation and Applications. New York, John Wiley \& Sons, 1986.

3. V e t s c h e r a, R. McView: An Integrated Graphical System to Support Multi-Attribute Decisions. - Decision Support Systems, Vol. 11, 1994, pp. 363-371. 
4. Ke e n e y, R., H. R a iff a. Decisions with Multiple Objectives. NewYork, John Wiley \& Sons, 1976.

5. V i n c k e, P. Multicriteria Decision Aid. NewYork, John Wiley \& Sons, 1992.

6. Ch a n k o n g, V., Y. H a i m e s. Multiobjective Decision Making: Theory and Methodology. North Holland, Dover Publications, 1983.

7. S p r a g u e, R., E. D. G a r s o n. Building Effective Decision Support System. Englewood Cliffs, New Jersey, Prentice Hall, 1982.

8. Ch in g-L a i, Hw an g, A. S. M. M a s u d. Multiple Objective Decision Making, Methods and Applications: A State-of-the-Art Survey. - In: Lecture Notes in Economics and Mathematical Systems. Vol. 164. Berlin, Heidelberg, New York, Springer-Verlag, 1979.

9. M i e t $\mathrm{t}$ i n e n, K. Nonlinear Multiobjective Optimization. New York, Springer Science+Business Media, 1999.

10. E hr g o t t, M., M. W i e c e k. Multiobjective Programming. - In: J. Figueira, S. Greco, and M. Ehrgott, Eds. Multiple Criteria Decision Analysis: State of the Art Surveys. London, SpringerVerlag, 2005.

11. We is troffer, H., S. Narula. The State of Multiple Criteria Decision Support Software. Annals of Operations Research, Vol. 72, 1997, pp. 299-313.

12. Zopounidis, C., M. Doumpos. Finclas: A Multicriteria Decision Support System for Financial Classification Problems. - In: Operational Tools in the Management of Financial Risks. C. Zopounidis, Ed. Boston, MA, Springer, 1998.

13. Kirilov, L. M., V. Guliashki, K. Genova, P. Zhivkov, B. Staykov, D. Vatov. Interactive Environment WebOptim for Solving Multiple-Objective Problems Using Scalarising and Evolutionary Approaches. - International Journal of Reasoning-Based Intelligent Systems, Vol. 7, 2015, No 1/2, pp. 4-15.

14. Kiri 1 o v, L. M., V. Gu li a sh ki, K. G e n ov a, M. V a s s i l e va, B. S t a y k o v. Generalized Scalarizing Model GENS in DSS WebOptim. - International Journal of Decision Support System Technology, Vol. 5, 2013, No 3, pp. 1-11.

15. Miettinen, K., M. M. Makela. Interactive Multiobjective Optimization System WWWNIMBUS on the Internet. - Computers \& Operations Research, Vol. 27, 2000, pp. 709-723.

16. G e g o v, A., D. S a n d e r s, B. V a t chova. Complexity Management Methodology for Fuzzy Systems with Feedforward Rule Bases. - International Journal of Knowledge-Based and Intelligent Engineering Systems, Vol. 19, 2015, No 2, pp. 83-95.

17. Collette, Y., P. S i arry. Multiobjective Optimization - Principles and Case Studies. Berlin, Heidelberg, Springer-Verlag, 2004.

18. S t a y k ov, B. Solving Multicriteria Optimization Problems with WebOptim Software System.Cybernetics and Information Technologies, Vol. 15, 2015, No 3, pp. 165-177.

19. A t a n a o va, T., N. B a k a n o va. Information Support for Decision Making in Organization Management. - In: Proc. of International Conference on Robotics, Automation and Mechatronics (RAM'14), 2014, pp.72-76.

20. I 1 i e v a, G. Group Decision Analysis with Interval Type-2 Fuzzy Numbers. - Cybernetics and Information Technologies, Vol. 17, 2017, No 1, pp. 31-44.

21. R a d e v a, I. Multicriteria Fuzzy Sets Application in Economic Clustering Problems. - Cybernetics and Information Technologies, Vol. 17, 2017, No 3, pp. 29-46.

Received 23.10.2017; Second Version 14.12.2017; Accepted 11.01.2018 